INPLASY

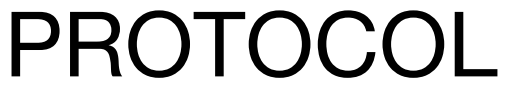

To cite: Rita et al. An update of the contemporary donor screening tests used in fecal microbiota transplantation for its future developments: $A$ systematic review. Inplasy protocol 2021120063. doi: 10.37766/inplasy2021.12.0063

Received: 13 December 2021

Published: 13 December 2021

Corresponding author: Margaret Ip

margaretip@cuhk.edu.hk

Author Affiliation:

Department of Microbiology, Faculty of Medicine, The

Chinese University of Hong

Kong.

Support: Seed Fund Ctr. Gut Micro. CUHK.

Review Stage at time of this submission: Data extraction.

Conflicts of interest:

None declared.

\section{An update of the contemporary donor screening tests used in fecal microbiota transplantation for its future developments: A systematic review}

Rita, WY Ng${ }^{1}$; Dharmaratne, P2; Margaret, Ip³.

Review question / Objective: Western pacific and South-east Asian region have its own lifestyle and dietary habits, for an example, the prevalence of parasites and the MDR pathogens are different compared to the European region where most consensus documents have been disseminated. Hence, current investigation is being carried out in view of appraising contemporary methods that have been used internationally and to propose rigorous donor screening methods appropriate for the regional requirement.

Information sources: EMBASE and MEDLINE through PubMed and WEB of SCIENCE. Additionally, we have reviewed all international consensus documents and local guidelines published in English.

INPLASY registration number: This protocol was registered with the International Platform of Registered Systematic Review and Meta-Analysis Protocols (INPLASY) on 13 December 2021 and was last updated on 13 December 2021 (registration number INPLASY2021120063).

\section{INTRODUCTION}

Review question / Objective: Western pacific and South-east Asian region have its own lifestyle and dietary habits, for an example, the prevalence of parasites and the MDR pathogens are different compared to the European region where most consensus documents have been disseminated. Hence, current investigation is being carried out in view of appraising contemporary methods that have been 
used internationally and to propose rigorous donor screening methods appropriate for the regional requirement.

Rationale: Lack of effectiveness of conventional antibiotics/treatments against gut disorders (gut dysbiosis), searching for an alternative therapeutic modality is warranted. Therefore, reshaping the gut microbiome (gut symbiosis) using FMT is an attractive strategy to restore appropriate microbiota homeostasis. In 2013, Van Nood et al. published the first randomized control trial, in which fecal material infusion was compared to standard treatment with vancomycin for recurrent CDI. Subsequent studies have also supported the efficacy of FMT over conventional treatment. Consequently, the growing interest of the scientific community in FMT has resulted in a large volume of data being published in the last decade. Despite several attempts on declaration of consensus documents on FMT protocol regionally as well as internationally, many issues require careful attention, such as appropriate donor screening procedures for safe and effective engraftment.

Condition being studied: Clostridiodes difficile infection (CDI) is defined by the presence of symptoms, usually diarrhea and either a stool test positive of $C$. difficile toxins or detection of toxigenic C. difficile, or colonoscopic or histopathologic findings revealing pseudomembranous colitis. C. difficile colitis results from the disturbance of the normal colonic flora, colonization of C. difficile and release of toxins causing mucosal inflammation and damage. Patients with multiple recurrences of CDI have failed to resolve their infection despite antibiotic agents targeting CDI are potential candidates of FMT. FMT has also been used in patients with severe refractory CDI. As a leading cause of morbidity and mortality, C. difficile is also an important cause of healthcare associated infection worldwide. The overall rate of health-care facility-associated $\mathrm{C}$. difficile infection rate was reported 2.24 per 1000 admission/year in a previous metaanalysis. In Hong Kong, the incidence of C. difficile has increased from 15.41 cases/ 100,000 persons in 2006 to 36.31 cases/ 100,000 persons in 2014 , an annual increase of $26 \%$. Inflammatory bowel disease (IBD), comprised of ulcerative colitis (UC) and Crohn's disease (CD), is a chronic, relapsing and remitting destructive disorder of the gastrointestinal tract. UC is characterized by the inflammation occurring in a continuous pattern in the colonic mucosa. In CD, there is transmural inflammation in a discontinuous distribution, involving any part of the GI tract. Etiology of IBD is not completely understood. Multiple factors may be involved, including dysregulated immune response to environmental factors, genetics and intestinal dysbiosis. Recently, FMT has been recognized as a potential treatment of this debilitating disease. The highest reported prevalence of inflammatory bowel disease were in Europe (ulcerative colitis 505 per 100000 in Norway; Crohn's disease 322 per 100000 in Germany) and North America (ulcerative colitis 286 per 100000 in the USA; Crohn's disease 319 per 100000 in Canada). Since 1990, the incidence has been rising in Africa, South America and Taiwan. The age-adjusted incidence of IBD per 100,000 individuals increased from 0.10 in 1985 to 3.12 in 2014 in a territory-wide populationbased study in Hong Kong. C. difficile is one of the most common causes of health care-associated infections (HAls). It is associated with substantial morbidity and mortality throughout the world and among people of all ages. In the United States, C. difficile caused an estimated half a million infections and 29000 deaths in 2012.

\section{METHODS}

Search strategy: A comprehensive search and systematic literature review was performed using 3 bibliographic databases namely, EMBASE and MEDLINE through PubMed and WEB of SCIENCE for the last decade, using the search terms, ("Faecal microbiota transplantation" OR "FMT" OR "bacteriotherapy" OR "gut microbiota transplantation" OR "fecal transplantation" OR "intestinal microbiota transfer") along with the term "AND" separately with 
("Clostridioides difficile" OR “C. difficile"), and ("Inflammatory bowel disease" OR "IBD" OR "Crohns's disease" OR "Ulcerative colitis" OR "UC"). The same search term was used in all databases to retrieve the data for this review.

Participant or population: The current study intended to review three major adult population (> 18 years) categories as (1) laboratory-confirmed diagnosis of $\mathrm{C}$. difficile colitis (severe or complicated CDI), (2) $>2$ laboratory-confirmed relapses of $C$. difficile colitis after receipt of initial specific antimicrobial treatment, (3) UC/IBD was diagnosed based on clinical, endoscopic, and histological criteria.

Intervention: The experiment group comprised with the individuals received single or multiple FMT regimens as an intervention. There will be no restriction in mode of delivery or either the status of the stool material.

Comparator: Since this is a qualitative analysis and the primary objective is to analyze donor screening procedures, the presence of a comparator is not applicable.

Study designs to be included: The review included all type of observational (retrospective and prospective) and clinical (randomized control, interventional, case and case series studies) studies on FMT against diversified gut related diseases as mentioned above.The systematic review will be included all type of clinical.

Eligibility criteria: The PICOS (Population, Intervention, Comparator, Outcomes, and Study Design) approach was used to guide the determination of the eligibility criteria (Amir-Behghadami and Janati, 2020) Population: The current study intended to review three major adult population (> 18 years) categories as (1) laboratoryconfirmed diagnosis of $\mathrm{C}$. difficile colitis (severe or complicated CDI), (2) >2 laboratory-confirmed relapses of $\mathbf{C}$. difficile colitis after receipt of initial specific antimicrobial treatment, (3) UC/IBD was diagnosed based on clinical, endoscopic, and histological criterialnterventions:
Single or multiple FMT in patients/ population with above underline medical conditions were included. All FMT administration routes were considered including; cecum through colonoscopy, oral gavage, naso-duodenal tube and delivered via enema. The fresh, frozen or encapsulated samples were used in FMT along with related or unrelated donors, were also included where, an adequate information was disseminated in donor screening procedure. Comparator: Since this is a qualitative analysis and the primary objective is to analyze donor screening procedures, the presence of a comparator is not applicable.Outcomes: Primary outcome of this review is to rationalize rigorous donor screening procedures suitable for South Asian region including to asses optimal strategy to detect ESBL due $t$ their high prevalence rates in the region.Study design: The review included all type of observational (retrospective and prospective) and clinical (randomized control, interventional, case and case series studies) studies on FMT against diversified gut related diseases as mentioned above.Excluding criteria: We excluded reviews, editorials, and letters to the editor or commentaries that did not provide precise and sufficient information for the donor screening analysis. Furthermore, the FMT applications for pediatric patients were also excluded due to inability to compare the outcomes with the adults stable gut microbiota. (Agans et al., 2011).

Information sources: EMBASE and MEDLINE through PubMed and WEB of SCIENCE. Additionally, we have reviewed all international consensus documents and local guidelines published in English.

Main outcome(s): Primary outcome of this review is to identify and appraise the existing methods in FMT donor screening procedures and to propose pivotal set of tests suits to South east Asia and westernpacific regions (according to the WHO classification) where high prevalence of multi-drug resistant (MDR) bacteria reported. 
Additional outcome(s): Appraise the commonly used donor screening test methods (PCR, serology etc.) used in FMT and comment the suitable test methods for the region of interest.

Quality assessment / Risk of bias analysis: Two researchers (RN, PD) independently assessed the eligibility of all articles and this was performed using the https:// www.covidence.org online platform. The article selection process included two sections. The titles and abstracts were reviewed firstly and then full-text articles were reviewed to judge the entitlement. Full texts that did not fulfill the priori defined inclusion criteria were excluded. Disagreements regarding inclusion in the final review were resolved through discussion with senior reviewer MI.

Strategy of data synthesis: Two researchers (RN and PD) developed an individual data abstraction spreadsheet using Excel version 2016 (Microsoft Corporation, Redmond, Washington, USA) and conducted the data abstraction for the included full-text articles and for the consensus documents. After which, the data were independently assessed by the review authors. We gathered the following information: type of study, time period, place of conduct, type of donor, donor screening timeline (where applicable), Questioner, list of blood and stool tests.

Subgroup analysis: Sub group analysis will be carried out for the type of donor screening procedures according to the WHO regional classification.

Sensitivity analysis: Since Meta-analysis is not involving, the sensitivity of the qualitative analysis will be assessed via $d$ discussion with the senior author to resolve disparities and final data set will be reviewed upon agreement of all authors.

Language: Yes, English.

Country(ies) involved: Hong Kong, SAR, China.
Keywords: Fecal Microbiota transplantation, Clostridioides difficile, Donor screening, IBD.

Dissemination plans: Peer-reviewed high impact factor journal.

Contributions of each author:

Author 1 - Rita WY Ng - Data screening, Data abstraction, critical revision and final approval of the article.

Email: ritang@cuhk.edu.hk

Author 2 - Priyanga Dharmaratne - Data screening, Data abstraction, writing the manuscript and final approval of the article. Email: priyanga@cuhk.edu.hk

Author 3 - Margaret Ip - Conceptualization, Critical revision and final approval of the article.

Email: margaretip@cuhk.edu.hk 